Trauma Berufskrankh 2008 10 [Suppl 1]:146-150

DOI 10.1007/s10039-008-1392-y

Online publiziert: 11. April 2008

(c) Springer Medizin Verlag 2008

\author{
H.-E. Schaller \\ Klinik für Hand-, Plastische, Rekonstruktive und Verbrennungschirurgie, \\ BG-Unfallklinik, Eberhard-Karls-Universität Tübingen, Tübingen
}

\title{
Infekte der Hand
}

Fremdkörperverletzungen können sogar unbemerkt bleiben oder sie werden nach einer scheinbar erfolgreichen Entfernung vergessen (- Abb. 1, 2). Oft kommt es zu Infektionen ohne oder mit sehr kleinen äußerlichen Defekten ohne wesentliche Funktionsausfälle und mit primär geringer Beschwerdesymptomatik. Daher schätzen sowohl Patienten als auch $u$. U. der primär behandelnde Arzt die Verletzung und die beginnende Infektion falsch ein und verursachen einen verspäteten Behandlungsbeginn.

\section{Diagnose}

Patienten, die sich mit so genannten Bagatellverletzungen an der Hand vorstellen, benötigen die gleiche anamnestische und diagnostische Abklärung wie Patienten mit größeren Verletzungen. Die eingehende Anamneseerhebung, Inspektion, Palpation und Funktionstestungen gehören ebenso zu dieser Abklärung wie die Labor- und Röntgendiagnostik.

Bei der Anamnese ist die Fragestellung nach der Schmerzlokalisation, die aber häufig nur ungenau angegeben werden kann, und Schmerzintensität von eminenter Bedeutung bezüglich der weiteren Indikationsstellung zur Operation. Ein pochender, die Nachtruhe störender Schmerz ist Ausdruck der Erhöhung des inneren Gewebedrucks im entsprechenden Kompartment der Hand und damit eine absolute Operationsindikation!

Auch bei der klinischen Untersuchung ist auf die Schmerzlokalisation, die fortgeleitete Schmerzsymptomatik (z. B. im Verlauf der Sehnenscheiden) und die schmerzbedingte Funktionsbeeinträchtigung (z. B. Schmerzen bei Beugung/Streckung oder Schonhaltung) größtes Augenmerk zu legen und bei Vorhanden- sein derartiger Beschwerden und Funktionsausfälle die sofortige Operationsindikation zu stellen. Die Art der Schwellung und Rötung, die Herabsetzung der Sensibilität und Beweglichkeit und/oder Fieber bestimmen in gleichem Maß die Indikationsstellung, das operative Vorgehen und die begleitende, u. U. nach dem chirurgischen Débridement einzuleitende antibiotische Therapie.

Die Röntgendiagnostik klärt über evtl. Fremdkörpereinsprengungen oder Osteolysen auf und bestimmt häufig genug das entsprechende operative Vorgehen (• Abb. 3).

\section{Therapie}

Die operative Planung und das operative Vorgehen erfolgen weit über die vermuteten Infektionsgrenzen hinaus bis ins gesunde Gewebe (• Abb. 6, 7). Dies gilt auch für die Wahl des anästhesistischen Verfahrens. Als Faustregel gilt hier, dass zwischen dem Infekt und der zu setzenden u. U. geplanten Leitungsanästhesie mindestens 2 Gelenke im Abstand zum Infektionsort gewählt werden. Der Eingriff wird in Oberarmblutsperre mit einer großzügigen Schnittführung nach handchirurgischen Prinzipien und mit mikrochirurgischen Methoden durchgeführt (Lupenbrille, Mikroskop, mikrochirurgisches Instrumentarium). Das Débridement sämtlichen eingeschmolzenen Gewebes erfolgt bis in gut durchblutete Strukturen ohne Rücksicht auf den Funktionsverlust und den hinterlassenen Defekt (- Abb. 8). Ein ausreichendes Débridement erfordert häufig genug einen plas-

Das Abstract dieses Vortrags wird regional im Abstractband des LVBG Thüringen veröffentlicht (ohne Bilder). 

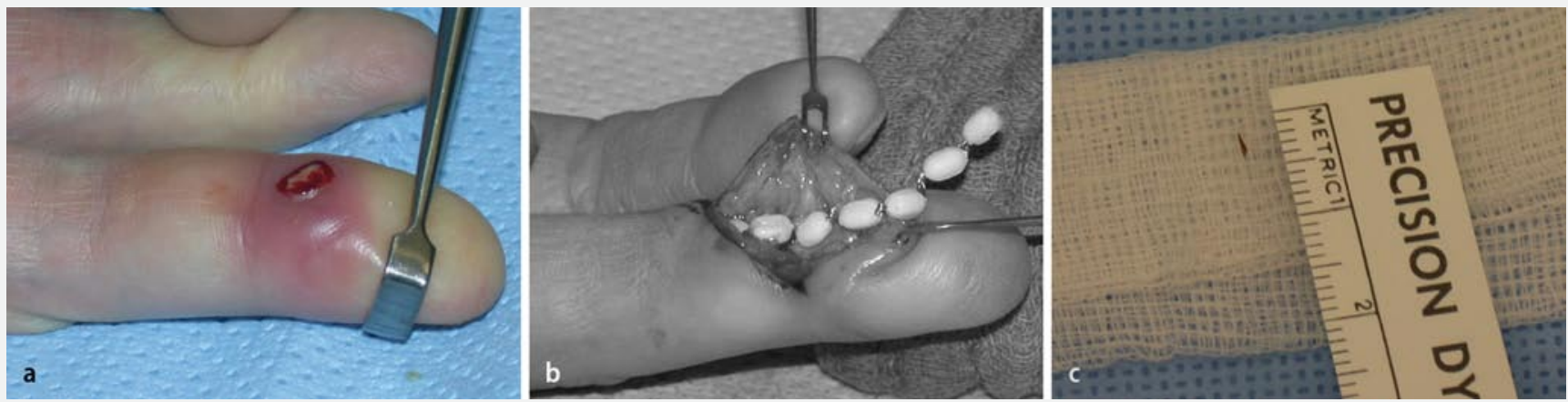

Abb. 1 \ Infektion nach Fremdkörperverletzung des Fingerendglieds, mögliche Ursachen: unbemerktes Eindringen, unvollständige Fremdkörperentfernung, a deutliche Rötung und Schwellung, b Débridement und Antibiotikakettentherapie, $\mathbf{c}$ entfernter Fremdkörper

Abb. 2 - Infektion des Fingermittelglieds nach Fremdkörperverletzung, a Klinik, b,c Fremdkörperentfernung und großzügiges Débridement, $\mathbf{d}$ entfernter Fremdkörper
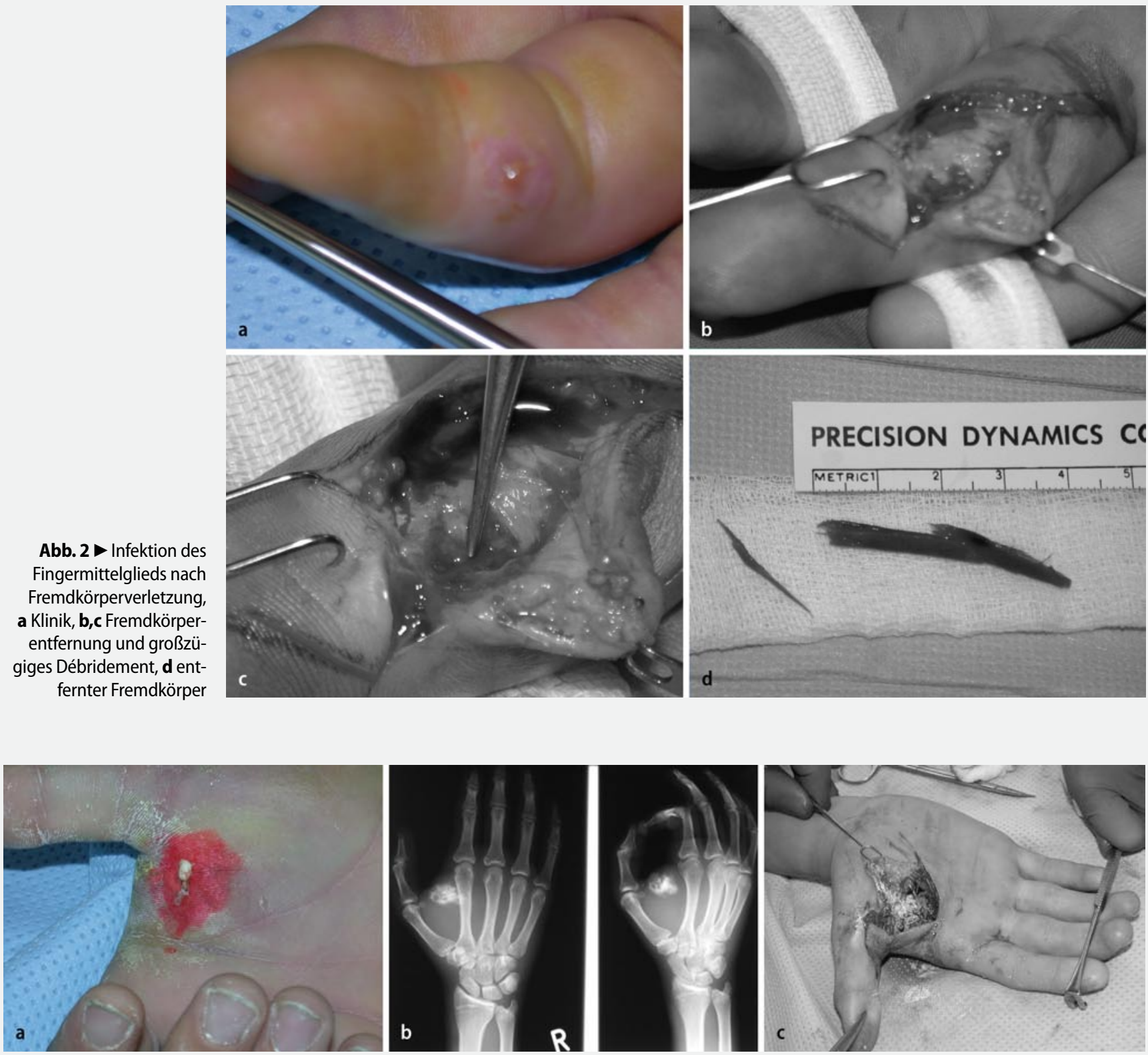

Abb. $3 \Delta$ Als Bagatellverletzung verkannte bzw. bagatellisierte und ohne Diagnose fehlbehandelte infizierte Hochdruckinjektionsverletzung, a Klinik, b Röntgen, c großzügiges Débridement 


\section{Handverletzungen}
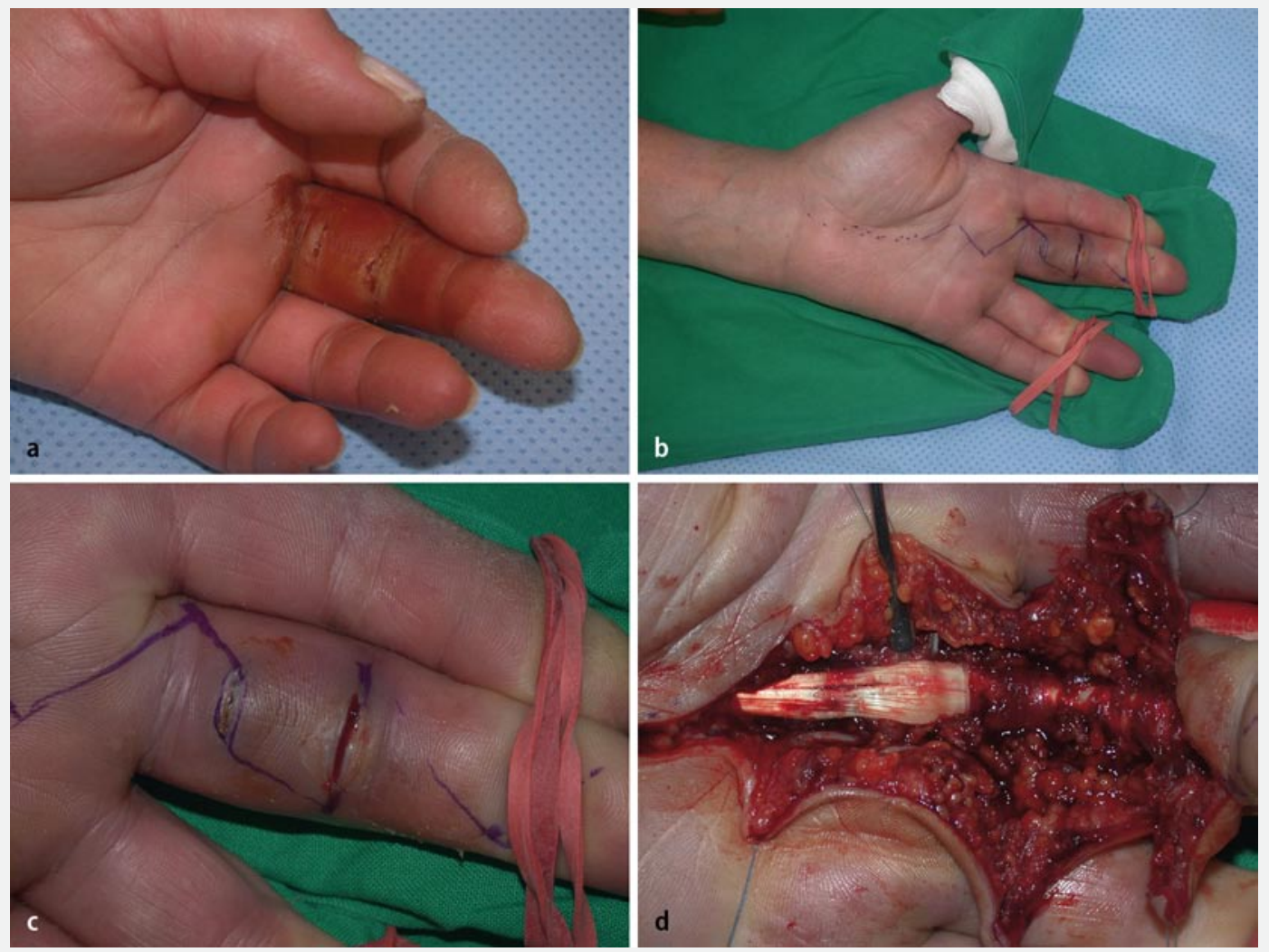

Abb. $4 \varangle$ Kammergrenzen überschreitende Infektausbreitung nach geringfügig erscheinender Primärverletzung des Mittelfingers, a Klinik, b,c Operationsplanung, $\mathbf{d}$ Operation
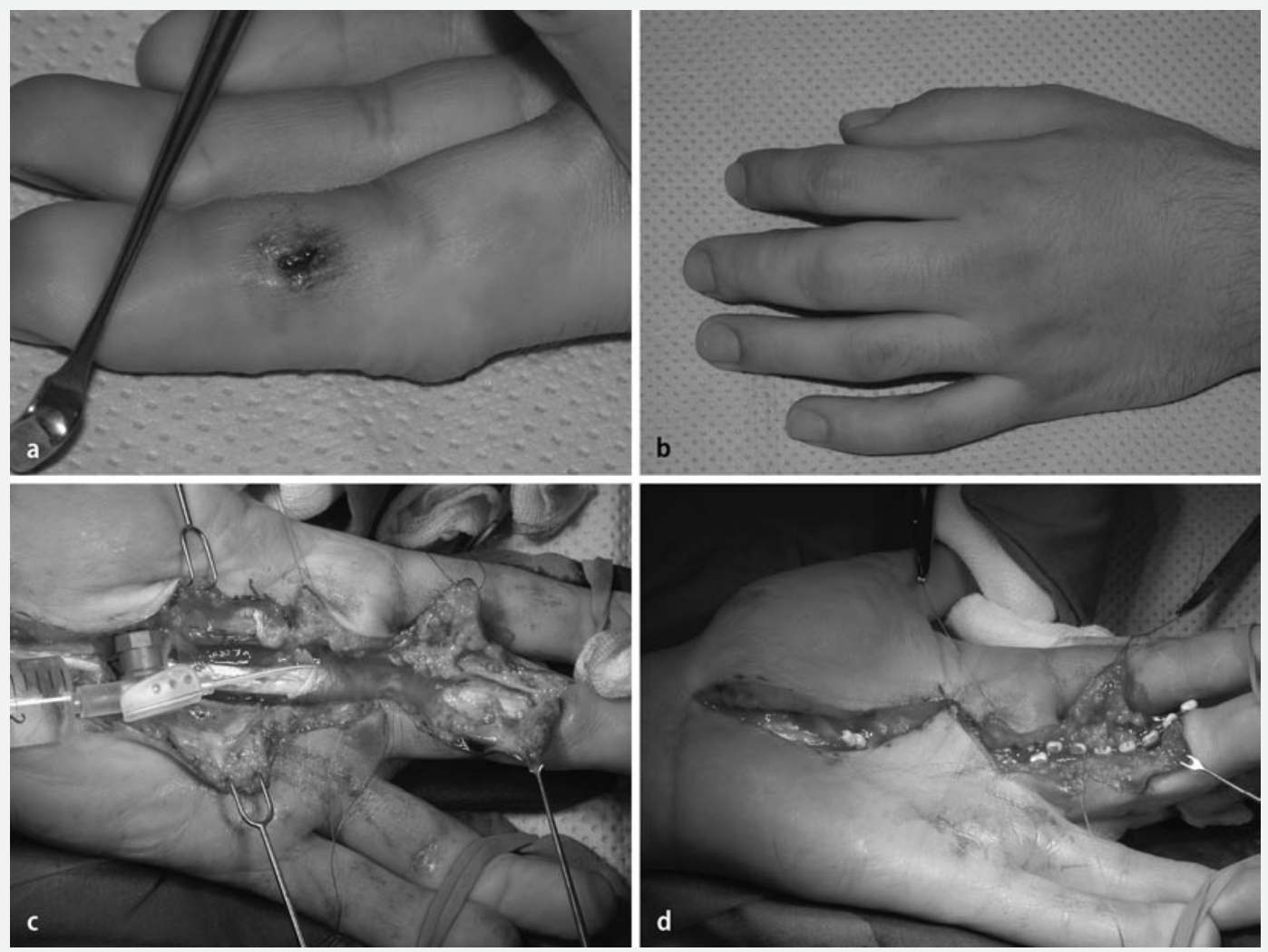

Abb. $5<$ Infektausbreitung im Kammersystem der Hand nach Verletzung des Mittelfingers, a infizierte Wunde, $\mathbf{b}$ deutliche Schwellung des betroffenen Fingers, c umfangreiches Débridement, $\mathbf{d}$ rekonstruktiver Defektverschluss 

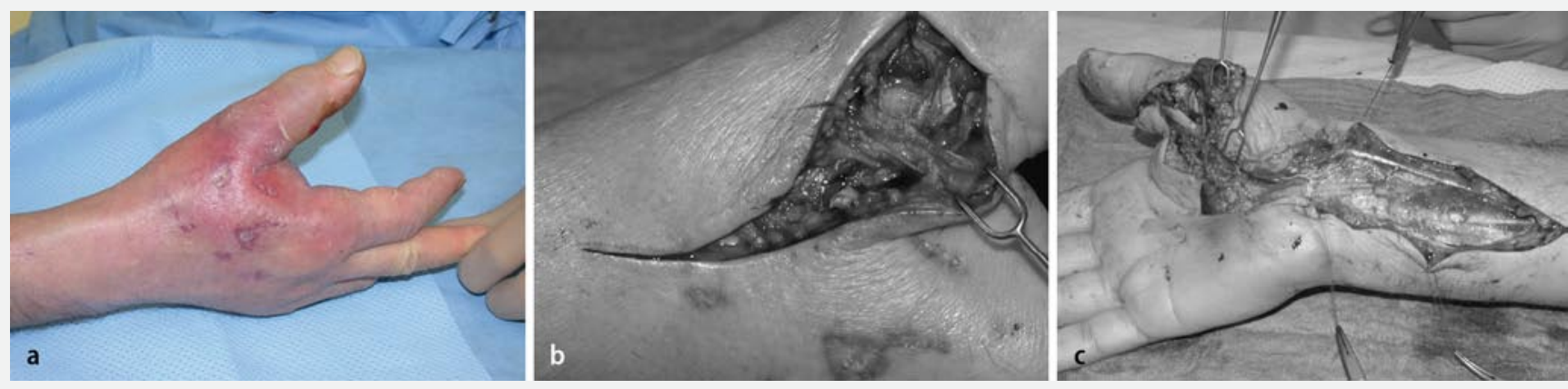

Abb. $6 \Delta$ Weit über die vermuteten Infektionsgrenzen hinausgehende Operationsplanung, a deutliche Schwellung und Rötung der infizierten Hand, b,c Débridement sämtlichen eingeschmolzenen Gewebes bis in gut durchblutete Strukturen
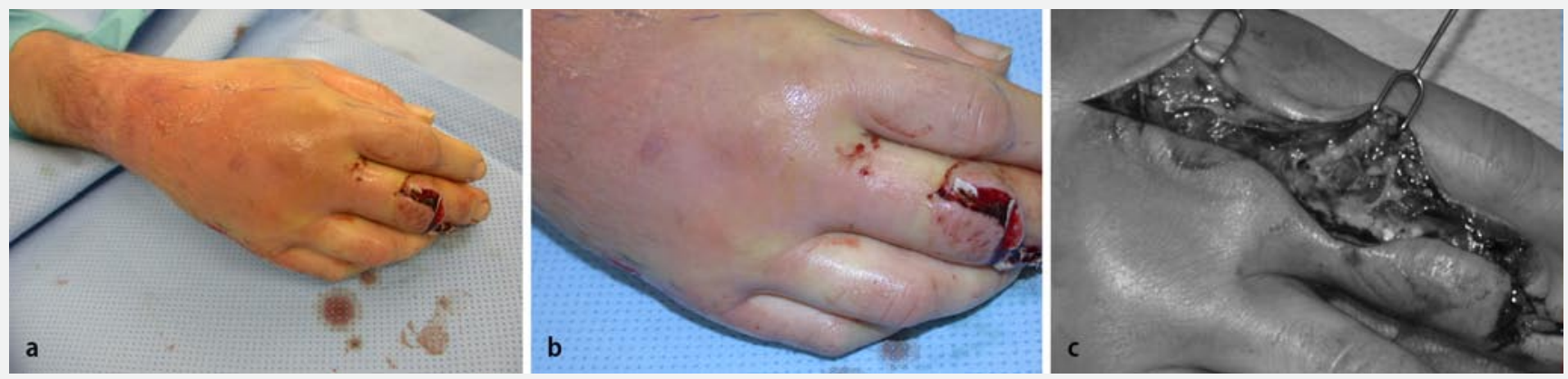

Abb. $7 \Delta$ Folgen einer ungenügenden prä- und intraoperativen Planung und Indikationsstellung (a,b) zum Revisionseingriff, beachte die Einstichstellen einer zu nahe am Infektionsort gesetzten Lokalanästhesie und die insuffiziente Schnittführung (c)
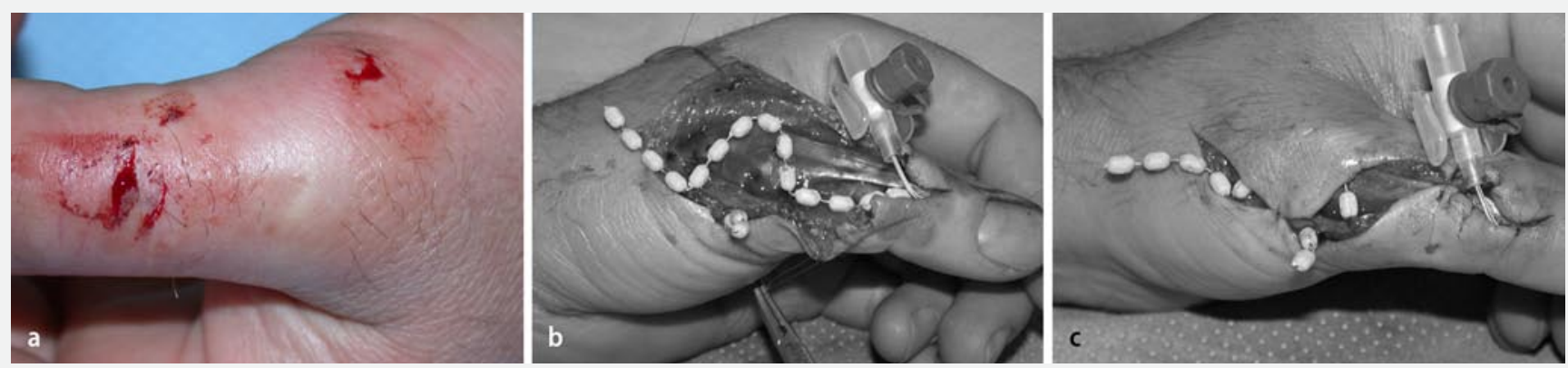

Abb. $8 \Delta$ Sofortige chirurgische Indikationsstellung (a), Débridement bis ins Gesunde (b) ohne Rücksicht auf Wundverschluss (c)

Abb. 9 Nach ausreichendem Débridement erforderlicher plastisch chirurgischer Defektverschluss, a infizierte Wunde, $\mathbf{b}-\mathbf{d}$ Ausheilungsergebnis nach Deckung durch heterodigitalen Insellappen vom streckseitigen Grundglied des Zeigefingers (Foucher)
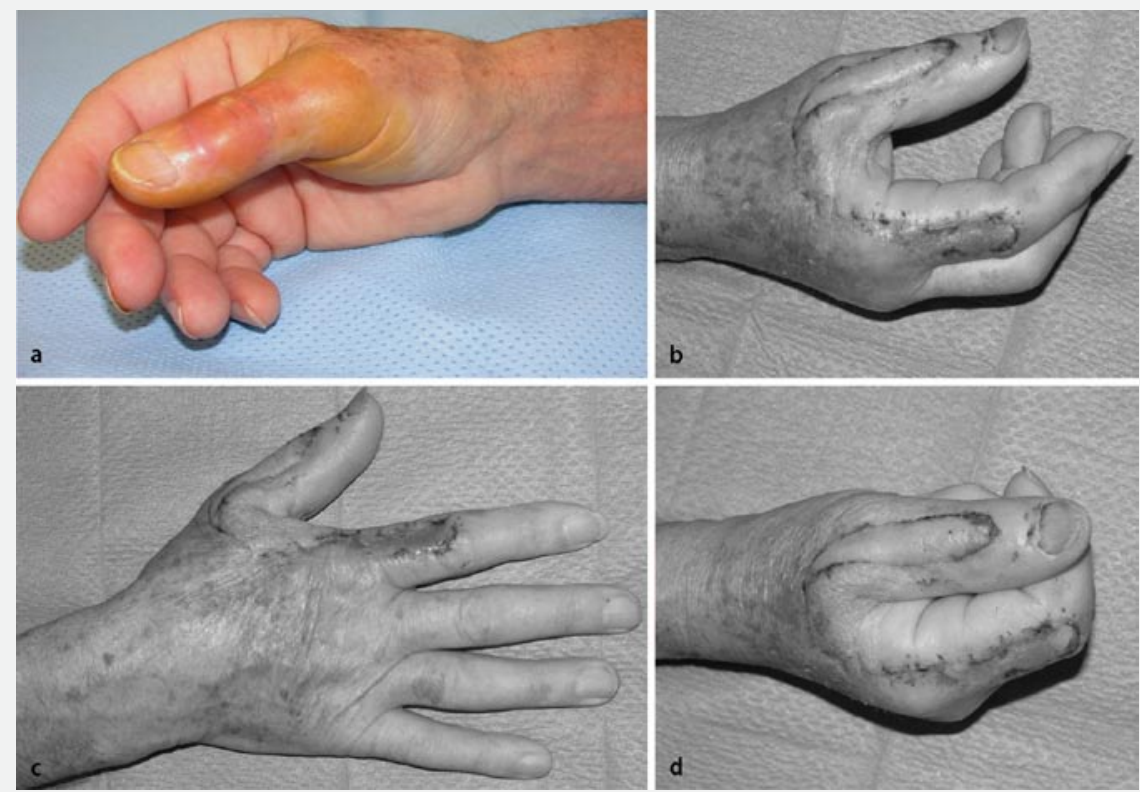
Trauma Berufskrankh 2008 · 10[Suppl 1]:146-150

(c) Springer Medizin Verlag 2008

\section{H.-E. Schaller \\ Infekte der Hand}

\section{Zusammenfassung}

Durch zu späte und/oder unzureichende chirurgische Intervention bei Handinfektionen kommt es zu langen Arbeitsunfähigkeitszeiten sowie schweren funktionellen Störungen der Hand. Ursächlich für das zu späte Eingreifen können eine Bagatellisierung von Verletzungen oder eine unsachgemäße Primärbehandlung sein. Um diese schweren Komplikationen nach Handverletzungen zu vermeiden, muss bei Patienten, die sich mit so genannten Bagatellverletzungen an der Hand vorstellen, die gleiche anamnestische/ diagnostische Abklärung wie bei Patienten mit größeren Verletzungen durchgeführt werden. Die operative Planung und Technik erfolgen weit über die vermuteten Infektionsgrenzen hinaus bis ins gesunde Gewebe

\section{Infections of the hand}

\begin{abstract}
Delayed or insufficient surgical intervention of hand infections can lead to long periods of being unfit for work as well as severe functional disabilities of the hand. The reasons that surgery is delayed for too long can be due to trivialization of injuries or incorrect primary treatment. In order to avoid such severe complications following hand injuries the same diagnostic procedure must be implemented including examination of the case history must be carried out with patients who present with so-called trivial hand injuries as with patients with severe injuries. The operative planning and techniques are carried out much further than the edges of the assumed infection limits far into the healthy tis-
\end{abstract}

ohne Rücksicht auf einen evtl. Funktionsverlust oder hinterlassenen Defekt. Häufig sind ein plastisch-chirurgischer Defektverschluss und andere rekonstruktive handchirurgische Maßnahmen erforderlich. Eine Antibiotikumbehandlung erfolgt nur nach ausreichendem Débridement und laut Antibiogramm. Neben ausreichender Ruhigstellung und strenger Bettruhe ist eine frühzeitige krankengymnastische, physio- und ergotherapeutische Mobilisierung zwingend erforderlich.

\section{Schlüsselwörter}

Handinfektion · Bagatellisierung .

Primärbehandlung · Débridement ·

Rekonstruktive Handchirurgie sue without consideration of possible functional loss or residual defects. Plastic surgery and other reconstructive surgical measures are often subsequently necessary to correct defects. Antibiotic treatment is only implemented after adequate débridement and according to an antibiogram. In addition to sufficient immobilization and strict bed rest, early exercise, physiotherapeutic and ergotherapeutic mobilization are absolutely necessary.

\section{Keywords}

Hand infection - Trivialize - Primary treatment · Débridement $\cdot$ Reconstructive hand surgery tisch-chirurgischen Defektverschluss (- Abb.9) und andere rekonstruktive handchirurgische Maßnahmen.

Die begleitende Gabe von Antibiotika ist nur nach erfolgtem Débridement und nach entsprechender Austestung indiziert. In der Regel erfolgt sie, wenn der Infekt nicht sicher beherrscht ist, weil die Grenzen der Infektion makroskopisch nicht klar bestimmbar sind, oder wenn Anhaltspunkte für eine septische Ausbreitung bestehen.

Nach dem operativen Primäreingriff erfolgt die großzügige Indikation $\mathrm{zu}$ einem Second-Look-Eingriff, falls erforderlich.

Neben ausreichender Ruhigstellung und der Anordnung strenger Bettruhe ist die Durchführung einer frühzeitigen krankengymnastischen, physio- und ergotherapeutischen Mobilisierung zwingend erforderlich.

\section{Korrespondenzadresse}

\section{Prof. Dr. H.-E. Schaller}

Klinik für Hand-, Plastische, Rekonstruktive und Verbrennungschirurgie, BG-Unfallklinik, Eberhard-Karls-Universität Tübingen, Schnarrenbergstraße 95, 72076 Tübingen eberhardschaller@gmx.de

Interessenkonflikt. Der korrespondierende Autor gibt an, dass kein Interessenkonflikt besteht.

\section{Literatur}

1. Schaller HE (1991) Handchirurgie, Mikrochirurgie, Plastische Chirurgie. S Reh Plass 23: 214-217 